\title{
RAILWAY INTERLOCKING PROCESS - BUILDING A BASE FOR FORMAL METHODS
}

\author{
Jacek Mocki, MIEAust MIRSE CPEng RPEQ \\ PhD Student, Griffith School of Engineering \\ Griffith University \\ Nathan campus, Griffith University, QLD 4111, Australia \\ jacek.mocki@griffithuni.edu.au
}

\begin{abstract}
Railway interlocking is designed to prevent trains from colliding. Generally speaking current systems are safe due to the processes that signalling systems have to follow as well as implementation procedures that interlockings are compliant with. Even the processes and procedures are very advanced; there are still train crashes that are triggered by interlocking faults. However, the interlocking faults are mainly related to poor maintenance of the systems or/and changes to the original designs without correct authorisation. While those areas are worth exploring to search some improvements it is also important that interlocking will progress its development toward intelligent transport system reinforcing maintenance functions.
\end{abstract}

Rail signalling systems went toward eliminating human factor by introducing machine based decision making on whether to proceed or stop. Development of electrical and electronic systems found interlocking moving toward relay solutions that became very common and are still used on railways. Finally computer based interlocking was introduced and successfully implemented in 90's.

Interlocking logic of those solutions has not been formalised so that it can be progressed toward intelligent transport systems. Interlocking functions are limited to ensure train operations are safe. Maintenance functions (e.g. fault prediction) or functions to enhance passengers' management are currently not implemented in rail interlockings.

This paper elaborates on currently implemented rail interlockings, compares various technologies implemented in those interlockings and proposes a base for consistent development of formal interlocking methods.

Keywords-railway; signalling; interlocking process; formal methods; railway control system

\section{FORMAL METHODS}

There is a number of literature articles [1-7] searching for a formal method to describe an interlocking. In those articles authors are making many assumptions trying to simplify railway infrastructure and train operations processes. The results of those formalisms are sometimes quite impressive; however, the unrealistic assumptions pushing it to be strictly scientific exercise that have very limited or no chance to be applied into real railway.

\author{
Dr Ljubo Vlacic, FIEAust FIET CPEng RPEQ CEng \\ Professor, Griffith School of Engineering \\ Director, Intelligent Control Systems Laboratory \\ Nathan campus, Griffith University, QLD 4111, Australia \\ l.vlacic@griffith.edu.au
}

Searching for a formal method to describe an interlocking it has to be understood how historically implemented interlockings look like and what processes have been followed in those interlockings.

Historical implementations of the interlockings will be analysed on an example of simple railway junction where a single bi-directional line diverts to the left. Therefore, there will be a turnout that requires changing its position to set the right route for a train. Also, some kind of indication to the train driver will be required to let the driver know there is no coming train from the other side and the route has been prepared for the train. The example of simple railway junction is reflected on Fig. 1.

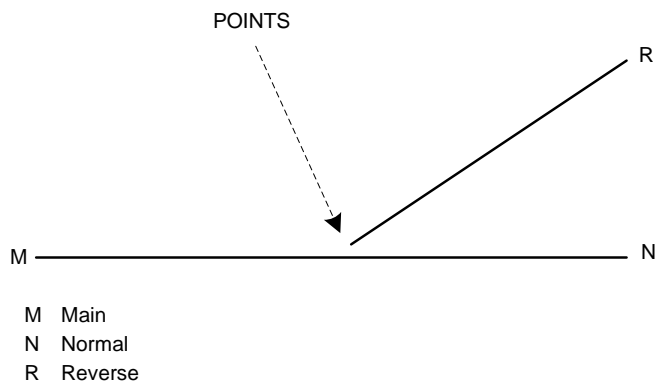

Fig. 1. An example of simple railway junction

No signalling equipment is presented on Fig. 1. The lines $\mathrm{M}-\mathrm{N}$ and $\mathrm{M}-\mathrm{R}$ represents railway track build on ballast.

It has to be noted that various types of rail, rail sleepers, ballast implemented and different gauges (distance between two rails) are used on railway. However, identical signalling interlockings can be built on various types of railway tracks.

Fig. 1 shows four possibilities for a train to travel through that railway junction: Main to Normal, Main to Reverse, Normal to Main and Reverse to Main. The points, therefore, have to be positioned Normal for the movements $\mathrm{M}->\mathrm{N}$ or $\mathrm{N}->\mathrm{M}$ and lied in Reverse when train is travelling $\mathrm{M}->\mathrm{R}$ or $\mathrm{R}->\mathrm{M}$.

In various applications the points are driven manually with a help of mechanical mechanism or electrically by means of points machine. 


\section{MANUAL (HUMAN) INTERLOCKING}

Since 1804 a high pressure steam's first locomotive hauled train has been publically demonstrated in Merthyr Tydfil (the UK). The steam era has revolutionised railway, however, it brought new problems into public. First passenger train death has been widely reported since September 1830. William Huskisson has been killed by Stephenson's Rocket at the opening of the Liverpool and Manchester Railway.

Since that death, the need for signalling became apparent. A railway first signalling system was recorded on 1832. At the beginning it was very simple not trivial however. The signalling logic relied on human that flag train with green flag for proceed signal and red for stop aspect.

A layout that shows a signalling arrangement for similar situation is reflected on Fig. 2.

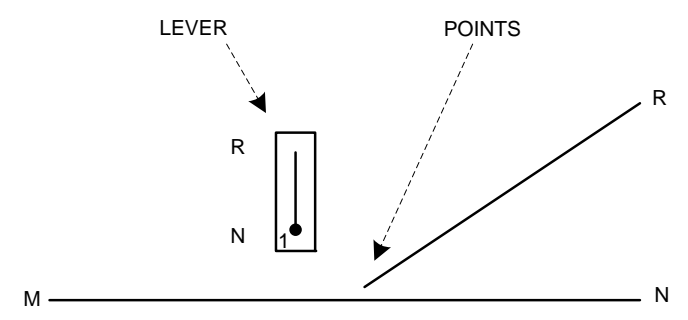

Fig. 2. A points operating lever - points in Normal position

The points on the picture are positioned Normal what is highlighted on the lever by a black dot next to number one. Number one tells the reader it is only one lever in this arrangement. In the case of a 4 lever ground frame it would be numbers 1, 2, 3 and 4 used. Letters $\mathrm{R}$ and $\mathrm{N}$ next to the lever show respectively reverse and normal position of the lever. Flagman is not shown on the picture.

Very first step in the manual interlocking process is to check what authority is given to the train. This is conducted by questioning if flagman raises the red flag to stop any train approaching the junction. If the red flag is not up route setting cannot be commenced, otherwise the interlocking enters a points position check.

There are two conditions in the points position check to be able to proceed to displaying proceed signal - train not passing the points and points in the correct position. Please note points position is judged by flagman. In the case train is on the set of points the interlocking process goes to the step Proceed train. If the points are required changing their position to Normal or to Reverse the interlocking processes to the state Moving points where flagman is physically relocating a lever associated with the points blades. Once points are moved flagman judges if the movement is finalised and if the points are locked properly. If not flagman is searching for any technical problems. Once technical problems are resolved flagman shall start the interlocking procedure again from the beginning.

When the points are positioned as required flagman decides to display a proceed signal. Proceed signal is given by raising a green flag up replacing the red flag. Obviously, interlocking process must account for any faults related to displaying green flag. It could be as simple as the flag is absent because flagman didn't check the bag with flags before going to site. Once the flag is in place the fault is obviously removed; however, flagman shall start the interlocking process from very first step again.

A train passage proceeds only when the green flag is properly displayed to the train driver.

Train passage is observed by flagman. Once the train passed the railway junction a process of route preparation can be initialised again. It could happen immediately after but it can take few days before a next train passes that railway junction. So, once flagman is on position there is a check for approaching trains and once again the interlocking process is followed.

\section{MECHANICAL INTERLOCKING}

Through the time of improving rail transport, signalling became more and more sophisticated, however, its basic rule remains - allow safe train operations meeting capacity demands. In this application first time signalling introduced term interlocking that is "an arrangement of signals and signal appliances so interconnected that their movements must succeed each other in proper sequence." [1] It wasn't actually signals; they were semaphores displaying a signal, one at the time. The signal appliances were mechanical points operated manually from a ground frame. The interconnection of the points and signals was designed in the ground frame and its operation was allowed only when unlocked by a key inserted into ground frame's lock mechanism.

This mechanical type of interlocking allows capacity of one train per 30-50 minutes depends on track arrangement, due to the fact that train route cannot be preselected and levers have to be manually operated. The life cycle of mechanical signalling is impressive. According to Mike Knutton (Senior Editorial Consultant) an average life for Mechanical locking frame in the UK is 75 years. [2]

\section{A. Mechanical points}

For mechanical points interlocking the railway junction layout on Fig. 1 is equipped in a 2 lever ground frame that is locked by an operator's lock. Points are installed with a points indicator. A layout that shows this signalling arrangement is reflected on Fig. 3.

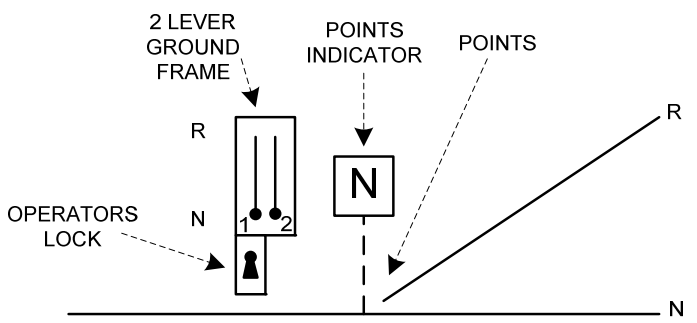

Fig. 3. Signalling arrangement for mechanical points interlocking

In this case, flagman is absent. An arrangement of different operator's keys within the corridors associated with the 
junction prevents situation that two trains approach the junction at the same time. Also, train driver holds the key. When approaching points, the points' indicator shows driver in what position the points are lying. If the position requires changing the train driver stops the train, turns operator's key in the ground frame and changes points position. Otherwise, train proceeds without stopping.

This type of interlocking is very sustainable and is still commonly used in for example, country side of New South Wales (Australia). However, the system has been enhanced with modern technology for train management using GPS tracking. GPS tracking is applicable only for railway lines with single track. For modern double, triple and more track arrangement more sophisticated signalling arrangement is desired.

Let's analyse interlocking process for this type of interlocking.

First condition in the process is to check the train authority. If train is not authorised to proceed interlocking process goes to its checking points position state. It could be that there is still a train passage. If this is the case the process enters Proceed train state, otherwise it searches correct points position.

When it is necessary to change points position an operator's key must be inserted into the operator's lock and turned to release a lever. For the example on Fig. 3, the operator's key unlocks ground frame release lever 1 .

There might be some problems in unlocking the lever. For example the operator's key is not suitable for the operator's lock. That would be actually very critical signalling system fault. Another example would be the key cannot be inserted due to dirty operator's lock mechanism. In all those cases the process enters Operator's key system fault state and returns to the initial state when the situation is rectified.

Once lever 1 is released it is moved to the opposite position releasing lever 2 and allowing for points position change. Also, in the case of any problems with lever 1 or/and lever 2 a ground frame fault state is introduced in the interlocking process. Analogically to operator's key fault the system is returning to its initial state when the problem is solved.

In the case of correct operation lever 2 is relocated to the end changing points position as required. The operator's key can be now turned and removed. Again, in the case of any technical problems the interlocking enters Operator's key system fault state and returns to the initial state when fault is removed.

Once the position change is finalised the interlocking process goes to the state of preparing route for approaching train and once the checks for points position are conducted an authority is given to the train. In the case of signalling arrangement on Fig. 3 it will be points indicator showing points in the right position and giving the authority to proceed. If a train management system with GPS tracking is built within this type of interlocking it could a radio communication authority given by a control centre. Once the authority is given the train passes the railway junction.

\section{B. Mechanical points and semaphores}

The interlocking example described in Section IIIA uses points indicator and train management system (if there is one installed). Mechanical points and semaphores interlocking use similar technology. The only difference is introduction of semaphores to give a train authority. A signalling arrangement for this type of interlocking for railway junction on Fig. 1 is presented on Fig. 4.

Three semaphores - two single (3 and 4) and one double (1, 2 ) are designed on the approach to the points 5. Double signal needs to be implemented due to operational requirements. Train driver needs to be informed if the train travels Normal or Reverse on the junction.

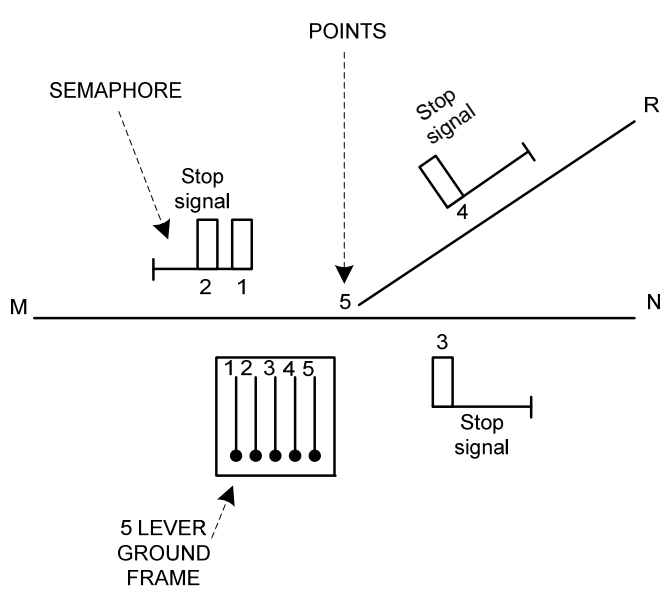

Fig. 4. Mechanical points and semaphores signalling arrangement

This arrangement influences application of 5 lever ground frame that is located close to the track. The semaphores as well as points are mechanically connected (set of rodding or/and wires) to the levers of the ground frames.

Ground frame has designed mechanical locking mechanism that allows only safe operation of the equipment and trains.

A drawing that shows locking mechanism for this arrangement is presented on Fig. 5.

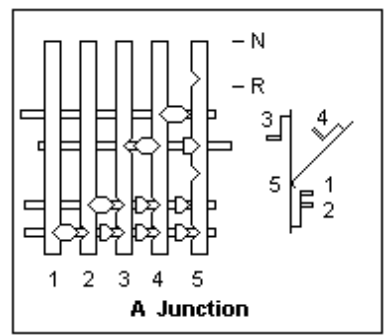

Fig. 5. Locking mechanism for 5 lever ground frame [6]

Similar to previously described process, the mechanical points and semaphores interlocking process begins with checking train proceed authority. Very first step of the process is to confirm that all arms of the semaphores are in horizontal positions. In the case position of arms is different to that expected the interlocking is detecting any semaphores system 
fault. If the semaphores are okay it means they are not in the horizontal position and the process is now back in the initial state waiting on finalising previous train operation control.

Once the semaphores are showing stop aspects the mechanical interlocking should let the lever 5 to be unlocked unless it experiences some technical issues that can be detected in this step and resolved in the state lever 5 system fault. When problem is removed the interlocking returns to the state unlock lever 5 .

A signalman visually checks points position. When signalman confirms absence of train on the points, it needs to be confirmed which route for train is being set. There are two possible choices - route $\mathrm{M}<->\mathrm{N}$ or route $\mathrm{M}<->\mathrm{R}$. If the points for route $\mathrm{M}<->\mathrm{N}$ are in reverse position the system requires a points position change. The signalman changes position of the lever 5 that relocates points to the normal position. Similar for the other route $\mathrm{M}<->\mathrm{R}$, if the points are in normal position lever 5 shall be operated to relocate points to the reverse position. Next part of the interlocking process is points position change, aspect display and train passage when setting routes $\mathrm{M}<->\mathrm{R}$.

The check of points position in this part of the process is to identify if the points are in correct position (post changing). The interlocking enters points movement system fault in the case of points or lever failure otherwise signalman checks which semaphore lever to pull to display the correct proceed aspect. If this is a route S2->R signalman pulls lever 2. Lever 4 is pulled when train approaching from semaphore S4 (route S4$>\mathrm{M})$.

In the next step signalman checks if the semaphore displays proceed aspect if not the system is in the state of failure for semaphore 2 or semaphore 4 . Otherwise train movement is authorised and train can proceed accordingly.

Then interlocking process waits on until train left the railway junction Once this is achieved signalman can start preparing another route obviously if there is a train approaching. Finally, the process returns to initial state.

Analogical scenario exists for routes $\mathrm{M}<->\mathrm{N}$.

\section{ELECTRICALLY POWERED INTERLOCKING}

The need for rail capacity and development of electricity pushes signalling to a next level. New lines are equipped in colour light signals instead of semaphores; the points are controlled electrically by points machines; interlocking is implemented in relay technology. The relay interlocking era has commenced since 1929.

Modern interlockings, those installed since late 1980s, are computer based interlockings (CBI). They have been implemented in many different configurations always taking under consideration latest computer developments. The earliest CBIs downsize the signalling rooms quite significantly. Development of those systems sees the interlocking being boxed in a microprocessor unit that size is as small as $\mathrm{H}: 200 \mathrm{~mm}, \mathrm{~W}: 50 \mathrm{~mm}$ and D: $200 \mathrm{~mm}$ while the relay system logic implemented in hardware takes space of one big bedroom.
Both types relay and computer based interlockings will be further analysed as electrically powered interlockings.

Railway junction from Fig. 1 has been signalled with colour light signals, points machine and train detection units. A layout for electrically powered interlocking is shown on Fig. 6.

In this particular scenario, control centre contains a control system and telecommunication equipment to be able to communicate bi-directionally with a signalling system that is located in SER building. PER building has only equipment needed to electrify signalling and telecommunication equipment located in SER, CER, location cases, colour light signals, points machine and train detection units. There is no telecommunication equipment in location cases. Interlocking as well as some train detection equipment is located in SER building.

Generally speaking, there are two types of electrically powered interlockings that can signal situation presented on Fig. 6 - relay interlocking and computer based interlocking.

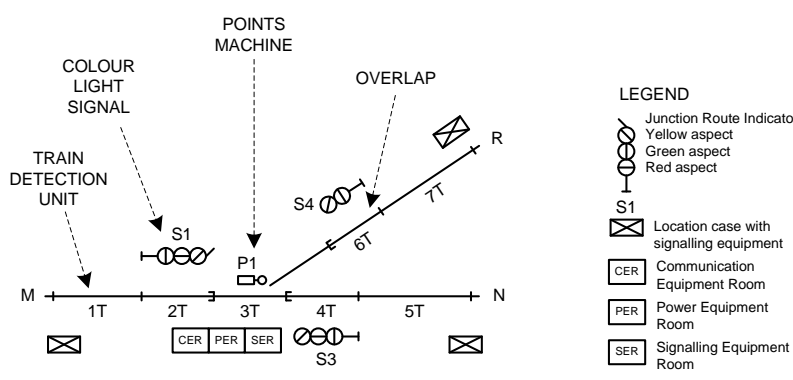

Fig. 6. Layout for electrically powered interlocking

\section{A. Relay interlocking}

Relay interlocking is built from a number of relays. The number depends on complexity of signalling layout being interlocked for a specific application. The types of relays used in the interlocking depend on functions being implemented as well as infrastructure managers' rules and relevant national standards. For example, relays used in Germany can be different to relays used in Australia; even relays used in the UK could be different to those used in Australia due to different National Electrical and Safety standards.

Also, within one country the relays could differ from installation to installation. However, the common, consistent point is the general rule of relay functioning as well as it's built (coil and contacts).

It can be observed in many relay rooms in Australia that Australian railway is using mostly $\mathrm{Q}$ relays that are mounted on a rack.

In terms of functionality, usually a single interlocking function is built from a single relay. However, relays made with two coils also exist in railway signalling. They are in one enclosure and effectively creating a relay that performs two 
functions. To simplify, it can be stated that one relay coil is performing one interlocking function.

Relay interlocking is designed in such ways that relay coils (functions) and relay contacts are wired in electrical circuits so that the entire interlocking will perform as a system specialised to the signalling layout. Therefore, the interlocking logic in this scenario is implemented by means of relay coils, contacts organised in electrical circuits that is performs functions in a parallel processing mode when contacts allow to do so.

\section{B. Computer based interlocking (CBI)}

Computer hardware (microprocessor, memory, hard drive, etc and input and output cards) and computer software (firmware, operational system and control programme) create CBI interlocking. Interlocking functions are defined in a control programme, also interlocking inputs and outputs and its association with the hardware are declared in the control programme. The control programme in the case of CBI is called interlocking logic.

In the instance of points machine, the computer interlocking is not capable to provide power to directly control points machine. Relays are still used to perform this functionality.

Computer hardware of CBI interlocking is different from a normal PC because the hardware must be specialised for safety related applications. Therefore, its construction is specific as well as operational system and firmware that are again different to a PC. However, CBI computers like PCs process information sequentially reading and executing instructions step by step in a clock cycles.

Interlocking logic is kept in a microprocessor module. This is a list of instruction predesigned specifically for a signalling layout and rules of operation (defined in control tables), checked, tested and compiled that can be performed by the interlocking hardware (computer) preventing trains from colliding.

List of instructions are prepared in graphical (contacts and coil like) or/and text (e.g. instruction if input $\mathrm{a}=0$ and input $\mathrm{b}=0$ then output $\mathrm{a}=1$ ) forms. The list of instructions can be stored in many files representing for example, definition of variables, inputs collection, internal instructions, and external instructions and outputs instructions. When compiled a one solid interlocking logic is created that is executable by interlocking hardware.

Hardware is controlled by firmware. Firmware is delivered in the hardware manufacturing process, is stored in one of the hardware components and can be changed only by the manufacturer. There is no railway signalling data kept in firmware.

\section{Relay and CBI - interlocking process}

Although Relay and Computer Based (CB) interlockings have technology and implementation differences from signalling design and railway operation perspectives there are many similarities that allow building a common process for those two interlocking types. Equipment used at trackside in both interlockings is practically the same, starting with motorised points and its detection, through colour light signals and finishing on train detection unit. Control panels in control centres are very similar. They can be implemented as electrically operated wooden panels painted to show signalling layout with small buttons and lights to allow control and indication and they can be computerised with signalling layout displayed on various screens with graphical, computer, representation for buttons and lights. Operator can control the buttons using a computer mouse.

There could be some cases where mechanical equipment is in use to control the railway through its relay interlocking. It is very unlikely that a mechanical control panel cooperates with a computer interlocking. Even if it was the rule to operate the computer interlocking would be similar to when cooperating with a relay interlocking.

Despite the various technologies designed in for the control panel as well as trackside equipment, the interlockings structure remains the same in all those cases.

The interlocking process prepared for relay and CB interlockings corresponds to the signalled railway junction shown on Fig. 6.

There are four possible routes for a train:

- from signal S1 toward signal S4 through reverse position of points $\mathrm{P} 1$, the route will be symbolled S1->R

- from signal S1 toward signal S3 through normal position of points $\mathrm{P} 1$, symbolled S1->N

- from signal S3 toward signal S1 through normal position of points $\mathrm{P} 1$, symbolled S3- $>\mathrm{M}$

- from signal S4 toward signal S1 through reverse position of points $\mathrm{P} 1$, symbolled S4->M.

The routes cannot be set at the same time because it would open the scenario for a train crash. Therefore, they have to be interlocked within each other allowing just one route at a time.

The system also will need to have an option to independently request individual points movement in a case where for example some maintenance work has to be conducted.

Very first step in the interlocking process is to check for any operator request and detect its kind. Then detect which exactly situation is being requested.

There are two possible types of operator request - points request and route request. When process identifies it is a points request it also detects if this is Reverse to Normal (R->N) request or Normal to Reverse $(\mathrm{N}->\mathrm{R})$ and initialising then the required points request.

In the case of route request it is identified which of the listed above four routes are requested. Additionally, it is checked whether the requested route is available. Once those checks are completed the interlocking starts a route preselection process. 


\section{1) Points request}

Points request is probably the simplest possible request. When initialising points request more checks is performed. Obviously, the request could be cancelled, so the process shall account for this situation. If the points request still goes ahead another check is to find out whether a route is being preselected or even locked. Finally, it has to be checked if points are positioned properly before the interlocking gives the command to start a points machine to change the points position. Points could be laid for example in Reverse position while request is from Normal to Reverse. The request is therefore invalid because the points are already in the requested position. Shall it happen a points request will be cancelled in this step checking also for any technical problems with the points. The technical problems could be related to points not being detected in either position.

In the process of moving the points there are two important elements - detecting points in requested position and detecting any faults that might occur when points are being moved.

Normally, it takes between 3 and 4 seconds to move a set of points (a points condition monitoring chart for Invensys's points machine M23A powered with $120 \mathrm{~V}$ DC is depicted on Fig. 7), however some points machines require more time to operate, for example where the same machine will use different motor and will be supplied with 24VDC (A chart for this scenario is presented on Fig. 8). In this case, it will take more than 10 seconds to finalise the points position change.

In the case where points operating properly and the movement is not finalised the interlocking will wait until the operation is completed. Once the points are detected in the correct position the process will jump to the state of waiting on another request.

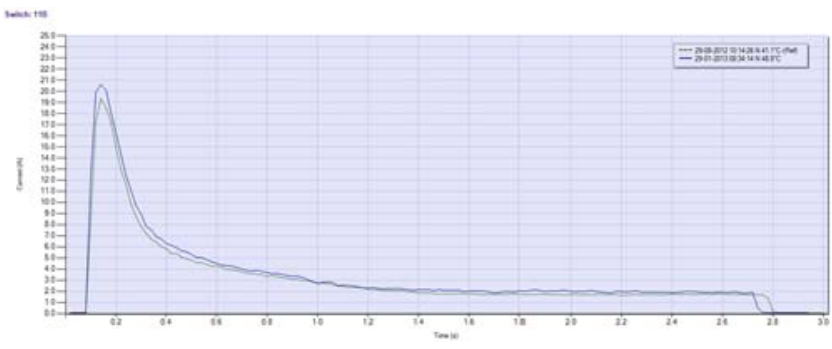

Fig. 7. A chart for Invensys's M23A points machine (operation N->R) [7]

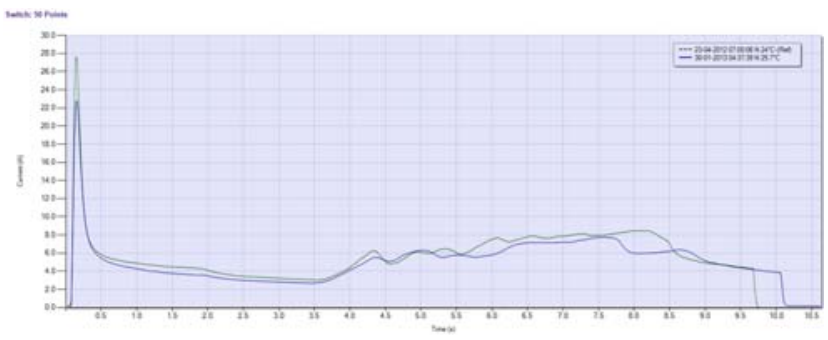

Fig. 8. A graph for M23A machine powered with 24V DC (operation N->R) [7]
When the control command has been initialised and points are moving incorrectly or not moving at all, interlocking will detect the situation and wait in this state until points are repaired and return to the state of checking train proceed authority waiting on another request.

\section{2) Route request}

Route request process is more complicated than just a points request. However, at some point points machine P1 is also involved. The route request process involves all trackside equipment.

It has to be acknowledged that all trackside equipment is vital for the interlocking; however, colour light signal is the medium to pass information to a train driver. So, interlocking must always remember to control the railway in such a way that in any unknown, unstable, undefined or intermediate state it has to display red aspect on a signal or signals related to that instability.

Very first step of route pre-selection is to search for any request cancellation. When route is cancelled this bit of the process forces interlocking to display red aspect on signal, in this case signal S1. Before deselecting the route interlocking looks at any technical problems with the signal. If a fault is identified the process keeps interlocking in a Red aspect fault state for as long as signal is fault free. When red aspect is displayed properly the route is deselected and interlocking returns to the initial state waiting on another request.

In the next step interlocking checks if any other conflicting routes are pre-selected. If yes the requested route is deselected and interlocking returns to the initial state. Otherwise it will start checking if signal displays red aspect and self-check the route pre-selection.

Once the route is pre-selected interlocking is looking for track sections that form the request route to be free (not occupied). By railway interlocking general rules it is prohibited to set a route if at least one track section being part of the route is occupied.

\section{3) Route setting}

Before commencing route setting it is vital to check once again for any route cancellation that may occur during implementation of a route request. In the case there is a cancellation, interlocking again enters the de-selection process described above. Otherwise, it commences route setting process.

In route setting process, it is essential to check points position and relocate them is the route requires so. For example, if points $\mathrm{P} 1$ are detected in Reverse position interlocking requires changing points position to Normal. If they are currently lying in Reverse position the interlocking steps to next task of route setting. Interlocking is searching for problems with points if points are not detected in Reverse and Normal position. Once points problems are rectified process returns to its pre-selection state. The reason being is that points repair may take some time and situation on track may change. A repair of extremely sophisticated points failures may take few days and in between repair activities trains operation can be controlled on site by a flagman. 


\section{4) Displaying proceed aspect}

The interlocking is now progressing to the last step before a train crosses the locked route. The step is to authorise the train to cross the section of track by displaying a proceed aspect.

Before proceed aspect is displayed there are two checks conducted.

Firstly interlocking confirms ability of the signal to display proceed aspect. When signal is not able to do so and a technical problem has been detected the interlocking enters a state of proceed aspect fault and stays in this state until the problem is solved then interlocking requires performing all checks for preselected route. More dangerous situation from train operation perspective is when interlocking does not control the signal, if proceed aspect cannot be displayed and no fault was recognised the process returns to its initial state informing the system about unrecognised state of proceed aspect on the signal.

Secondly, interlocking searches for any route cancellation that is still possible at this stage. In the case of route request cancellation interlocking returns to the initial state through displaying Red aspect state described above in pre-selection process. Otherwise the interlocking allows to display proceed aspect on the signal.

Once the command was given and trackside equipment processed the request it is expected that signal provides train driver with authority to proceed to the next track section. Interlocking checks if the signal has been displayed properly progressing to a state of displaying Red aspect when the signal failed to do so. Interlocking also commences Red aspect display in the case of route request cancellation.

In the state of displaying Red aspect after a proceed aspect command has been introduced, interlocking seeks any technical problems with the signal and if problems are removed interlocking enters Release approach locking state. This is very important state that is strictly related to train operation. Approach locking function keeps the route locked for any train approaching the signal. Once released the function looks back through train detection units occupancy if the route can be immediately released. If a section or sections of track are occupied the route is released at certain conditions through a time. The conditions and the time are defined in control tables specifically designed for a signalling arrangement. The example on Fig. 6 does show only one section of track in the front of signal S1. Therefore, in this example interlocking looks only at this bit of track for route S1->N, however, in more complicated layouts approach locking can be very sophisticated.

\section{5) Train passage and route release}

Final bit of the interlocking process is to look for the train passage and release the route allowing another route request.

Looking at the signalling principles when the first axle of a train crosses a section of track behind a signal the signal must immediately display Red aspect. Looking at the Train operated route release of the interlocking, it can be observed that for route $\mathrm{S} 1->\mathrm{N}$ the interlocking searches for occupancy of the train detection unit $2 \mathrm{~T}$. Immediately after the track $2 \mathrm{~T}$ enters the occupation state the process forces Red aspect on signal S1 checking then if the red aspect is properly displayed. Once the red aspect is properly detected interlocking is waiting on train passage and release of all track sections involved in route S1$>\mathrm{N}$. Unlocking the route and return to the initial state commences straight after train detection units confirmed the track sections are not occupied.

\section{Implementation differences}

The relay interlocking influences the need to control trains remotely due to the fact that interlocking is now centralised for quite extensive area and human actions are limited to requesting route pre-selection. Interlocking logic in this case is implemented in circuits that are performing specific functions. Interlocking in these circuits is done by relay contacts from relays performing those specific functions.

Relays offering the following advantages:

- low technical risk regarding safety approval

- operational performance that can meet all requirements, and

- an economic life long enough to match the capacity of the industry to maintain and renew installations in the long term. [2]

Analysing the process for Relay and Computer Based interlockings (Section IVC) under implementation in relay technology it has to be noted that many operations within sequential process of the interlocking is conducted by parallel processing of relay operations. For example conditional steps of the interlocking are reacting on inputs from trackside equipment. If train gets authority to proceed and commences its journey entering next track section the relay function for the track section immediately informs the system the train is on. Contacts of that relay are in the colour light signal; therefore it reacts to that track section occupancy displaying red aspect. Those actions are not waiting on interlocking process. So, the relay interlocking has two kinds of processing: sequential interlocking process and parallel interlocking functions implementation. Sequential processing of relay interlocking is done by implementing a hierarchy of relay circuits. Effectively appropriate arrangement of relay contacts in those circuits provides sequence while relays are concurrently processed.

Also important is the relay interlocking life cycle and capacity. According to Mike Knutton, the average life cycle of electro-mechanical relay in the UK is 25 years. [2] The relay interlocking allows capacity of one train every 3-5 minutes again depending on track arrangement.

Modern interlockings, those installed since late 1980s, are computer based interlockings (CBI). They have been implemented in many different configurations always taking under consideration latest computer developments. The earliest CBIs downsize the signalling rooms quite significantly. Development of those systems sees the interlocking being implemented in a microprocessor unit that size is as small as H: 200mm, W:50mm and D: 200mm while the relay system logic implemented in hardware takes space of one big bedroom or more. 
There are few reasons why the CBI interlockings are introduced to railway in the space previously occupied by relays. They are following:

- interlocking logic previously implemented in circuits (hardware) is done in computer programme (software) that is run in computer hardware

- computer hardware necessary to implement an interlocking takes much less space than its equivalent in relays

- interlocking can be installed remotely and be transmitted to/from the objects (signal, points machines, etc)

- electronic implementation of interlocking allows digital or software communication with other systems (passenger information system, maintenance system, etc).

Comparing the technology implementation in greater details computer based interlocking is processed sequentially in computing processing. Additionally, conditional checks are also implemented in computing processing. Obviously in this type of processing it is required to have some kind of operational system and some firmware. Both elements are also computed in the same process what create the interlocking process being much longer. In terms of detailed processing it has been observed that computers in some interlocking implementation (for complicated stations) are processing slower than an equivalent in relay technology.

The capacity that CBI interlocking provides is similar to relay interlocking can offer. It has been observed that the CBI logic processing time is generally slower than relay interlocking is.

There are many advantages and disadvantages of computer based interlockings. However, signalling experts are not convinced which interlocking implementation gives more benefits or simply which technology suits better current railway needs. According to Mike Knutton (Senior Editorial Consultant), unforeseen delays in the acceptance process for computer-based interlockings (CBI) in Britain has persuaded at least some experts that relay interlocking technology still has a valid and developing future for controlling line-side signalling. [2]

Those unforeseen delays in the acceptance process for CBI can be caused by the following:

- lack of formal methods to describe behaviour of the interlocking

- many different applications of CBI interlocking

- railway expectations to implement more functionality at the same interlocking processing speed

- tools to maintain CBI interlockings are not efficient or there is lack of such tools

- estimated average life of equipment.

\section{CONCLUSIONS}

In this paper three main types of existing railway interlockings have been described for the simplest possible railway junction (Fig. 1) signalled with a signalman, semaphores and colour light signals.

Relay and computer based interlockings have been compared at high level under technology implementation.

The following conclusions can be made after researching those three types of interlockings:

- This paper is documenting interlocking processes and trying to categorise current interlocking implementations for a simple railway junction. This is still necessary to conduct similar study on more complicated layouts to be able to confirm consistency between interlocking process in each of those types of interlockings

- Currently implemented interlockings are constrained to perform only interlocking functions related to the train operations ensuring those operations are safe. Intelligent transport features are not implemented due to critical processing time. It is understood that relay interlocking system design was performed long time ago where intelligent transport definition was absent. CBI processing is slow and introduction of new functions can only interrupt in standard interlocking functions computing

- All types of interlocking have sequential interlocking process. Wherever new technology or/and concurrent interlocking processing are considered there will be always steps in the interlocking process and therefore some sequential process necessary. It seems to be desired to combine advantages of relay and computer based interlockings when seeking new interlocking process and new technology implementation.

\section{REFERENCES}

[1] Dictionary definition http://interlocking.askdefine.com/

[2] Mike Knutton, "Back To The Future With Relay Interlockings"; International Railway Journal; December 2003

[3] Institution of Railway Signal Engineers, "IRSE Top Inventions - Vote Now!”, IRSE News, Issue 182, October 2012

[4] Eugenio Roanes-Lozano, Antonio Hernando, Jose Antonio Alonso, Luis M. Laita, "A logic approach to decision taking in a railway interlocking system using Maple", Mathematics and computers in simulation 82 (2011) pages 15-28

[5] Jacek Mocki, "Confirmation of Candidature Report - The concurrent processing of railway interlocking logic”. Griffith University, Australia 2009

[6] http://mysite.du.edu/ etuttle/rail/lock.htm, Railways History, Signalling, Engineering website

[7] Points Condition Monitoring by Strukton (www.strukton.nl), printscreens from real Queensland and Western Australia implementations of the system monitoring Invensys Rail points machines M23A 